Neurosurg Focus 15 (2):Article 14, 2003, Click here to return to Table of Contents

\title{
Benign sacral tumors
}

\author{
Harel Deutsch, M.D., Praveen V. Mummaneni, M.D., Regis W. Haid, M.D., \\ Gerald E. RoDTS, M.D. AND STEPHEN L. ONDRA, M.D.
}

\begin{abstract}
Primary tumors of the sacrum are rare. In adults, the most common sacral tumors are metastases. The most common primary sacral tumor is a chordoma. Chordomas along as well as tumors such as chondrosarcomas, osteosarcomas, myxopapillary ependymomas, myelomas, and Ewing sarcomas are considered malignant. In this article the authors focus on benign sacral tumors.
\end{abstract}

KEY WORDS • sacrum • tumor

The sacral compartment contains two distinct tissues from which primary sacral tumors may arise: bone and neural elements. ${ }^{6}$ In this article, we will focus only on benign sacral tumors arising from these two tissues.

Benign sacral tumors can grow to very large sizes before producing symptoms. Tumors involving the upper sacrum can cause gastrocnemius weakness by compressing the S-1 nerve root. Patients with lower-region sacral tumors tend to present with perineal numbness, loss of bowel and bladder control, sexual dysfunction, and pain. Occasionally, however, these tumors can be asymptomatic and grow large enough for their anterior portion to be palpated during a rectal examination.

\section{BENIGN SACRAL TUMORS IN CHILDREN}

In children, the most common sacral tumors are SCTs. ${ }^{18}$ These lesions are congenital and usually benign. Approximately $20 \%$ of cases are identified prenatally, and $70 \%$ are identified at birth. The remaining $10 \%$ are identified within the 1st year of life and usually present with intestinal obstruction. Histologically SCT possess benign mature characteristics in $70 \%$ of cases. ${ }^{21}$ The remaining $30 \%$ have histologically malignant features typical of either immature teratomas $(20 \%)$ or endodermal sinus tumors $(10 \%)$. Most SCT resections are performed via a posterior approach, although in one third of the cases a combined abdominal-sacral approach is required. There is a $10 \%$ recurrence rate after 5 years in patients harboring mature teratomas. When these tumors recur, the histological examination may reveal either mature teratomas or endodermal sinus tumors. Serial serum $\alpha$-fetoprotein measurements may be used to monitor for tumor recurrences.

Abbreviations used in this paper: $\mathrm{ABC}=$ aneurysmal bone cyst; $\mathrm{CT}=$ computerized tomography; GCT = giant cell tumor; GSS = giant sacral schwannoma; $\mathrm{MR}=$ magnetic resonance; $\mathrm{SCT}=$ sacrococcygeal teratoma.
Other very rare benign sacral tumors in children include lipomas, dermoids, and epidermoids. ${ }^{9}$ Congenital abnormalities such as spina bifida occulta, hairy nevi, dermal sinus tracts, and dimples are associated with epidermoids, dermoids, and teratomas. Associated anomalies such as tethered cord need to be assessed in these patients. ${ }^{12}$ These tumors often enlarge significantly before detection.

\section{BENIGN SACRAL TUMORS IN ADULTS}

\section{Bone Tumors}

Aneurysmal Bone Cysts. Aneurysmal bone cysts represent $1 \%$ of all primary bone tumors, and $3 \%$ of $\mathrm{ABCs}$ arise from the sacrum. ${ }^{15}$ These tumors are benign, and patients typically present with mass effect or pain.

Radiographically, ABCs typically appear osteolytic. Computerized tomography scanning demonstrates multiple fluid-fluid levels within cystic cavities in the bone. Magnetic resonance imaging reveals multiple fluid-fluid levels, and the lesions are typically nonhomogeneous. A low signal rim usually encircles the cystic lesion on $\mathrm{T}_{1}$ and $\mathrm{T}_{2}$-weighted MR imaging sequences.

On gross examination, an ABC is a blood-filled cavity with a thin periosteal outer membrane. Soft fibrous walls separate spaces filled with blood clots. Microscopically, the $\mathrm{ABC}$ has cystic spaces filled with blood. Left untreated, these tumors have an indolent course. Our preferred method of treatment is excision. Other surgeons have performed intralesional curettage and endovascular therapy. ${ }^{4}$ Aneurysmal bone cysts may recur after excision, however, and intralesional curettage alone is associated with a high recurrence rate. ${ }^{13}$

Papagelopoulos, et al., ${ }^{14}$ reported recurrence of $\mathrm{ABC}$ in five $(14 \%)$ of 35 patients treated at the Mayo Clinic. The lesions were generally removed by intralesional resection. Most recurrences occurred within 18 months of treatment. ${ }^{14}$ Untreated, these tumors have an indolent course. ${ }^{13}$ Some authors have advocated endovascular therapy either as a stand-alone treatment or in conjunction with surgery. ${ }^{4}$ 
Giant Cell Tumors. The incidence of GCTs peaks is in the third decade of life. They represent approximately $20 \%$ of all benign bone tumors. Even histologically benign tumors, however, can metastasize to the lung, and 5\% of patients with GCTs have lung metastases.

Giant cell sacral tumors appear as a radiolucent sacral lesion on radiography. Computerized tomography scanning demonstrates a soft-tissue attenuation with distinct margins and occasional surrounding sclerosis. The MR imaging-based appearance can be variable with a heterogeneous low-intensity image on $\mathrm{T}_{1}$ - and $\mathrm{T}_{2}$-weighted sequences.

Giant cell tumors have a distinct histological appearance comprising with multinucleated osteoclastic giant cells intermixed throughout a spindle cell stroma.

Treatment for sacral GCTs is similar to ABCs. If complete resection cannot be achieved easily, intralesional curettage is possible, although it is associated with a high recurrence rate. In a review of 26 cases at the Mayo Clinic, Turcotte, et al., ${ }^{20}$ reported a 33\% recurrence rate. Some authors have advocated cryosurgery as an intraoperative adjuvant. ${ }^{11}$ Like ABCs, endovascular therapy is also an option. Tumors may be embolized by placing coils and gelfoam particles. Postoperatively CT scanning can demonstrate some reossifications, and angiography reveals decreased vascularity. Lin, et al., ${ }^{10}$ demonstrated immediate symptomatic improvement in 14 of 18 patients in whom embolization was performed. Of the successfully treated patients, tumor growth or symptomatic recurrence occurred in $43 \%$ at 15 years. $^{10}$

Osteoid Osteomas. Osteoid osteomas represent approximately $10 \%$ of benign bone tumors. They are rarely found in the sacrum. ${ }^{3}$ Osteoid osteomas commonly affect adolescents. Patients classically present with nocturnal pain located in the sacral area. The pain is also often relieved by salicylates.

On CT scans, osteoid osteomas appear as a sharp round or oval lesion that is less than $2 \mathrm{~cm}$ in diameter, has a homogeneous dense center, and has a 1 to $2-\mathrm{mm}$ peripheral radiolucent zone (Fig. 1). Radionuclide scanning for ${ }^{99} \mathrm{Tc}$ diphosphonate demonstrates intense radioisotope uptake.

Histological examination shows a nidus consisting of a combination of osteoid and woven bone surrounded by osteoblasts. The nidus is surrounded by sclerotic bone.

En bloc resection is usually possible and the recurrence rate is low (Figs. 2 and 3). ${ }^{2}$

\section{Neural Tumors}

Giant Sacral Schwannomas. The most common neuralderived benign sacral tumor is the GSS. These lesions exhibit a characteristic radiographic appearance. ${ }^{16}$ On MR imaging, GSS typically appears as a large, well-defined, heterogeneous presacral soft-tissue mass with a low signal rim, arising just to one side of the midline, with minor underlying bone erosion. The GSS is predominantly solid, but cystic areas may be observed on $\mathrm{T}_{2}$-weighted images. Their mean diameter is approximately $10.5 \mathrm{~cm}$. There is often anterior displacement of the pelvic organs but usually evidence of invasion. Cyst formation, hemorrhage, and necrosis are relatively common in GSS. These lesions also tend to be encapsulated (unlike neurofibromas). Most can be resected completely, and recurrence is rare. ${ }^{1,15}$

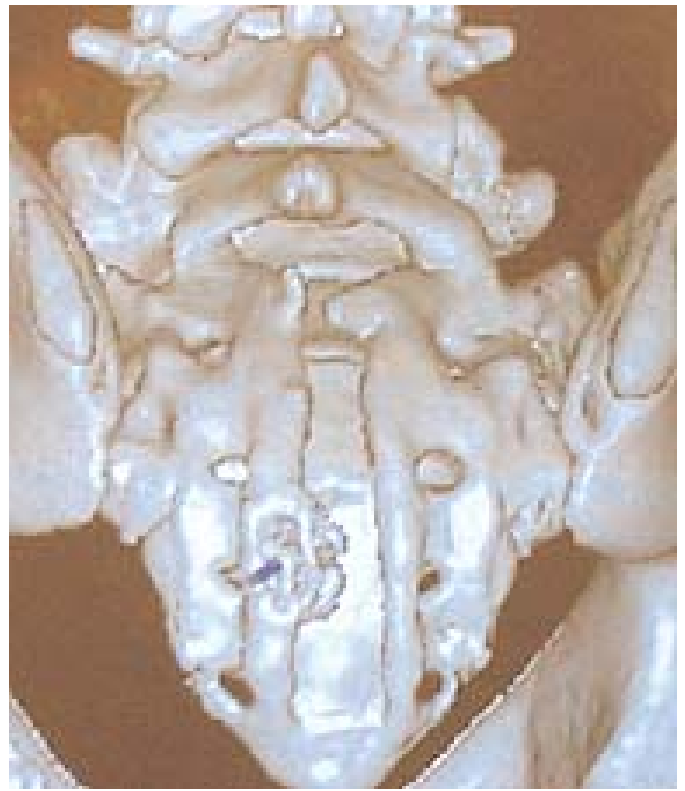

Fig. 1. Preoperative three-dimensional CT reconstruction demonstrating a sacral osteoid osteoma in a child. Note the small wellcircumscribed lesion.

\section{SURGERICAL TREATMENT OF BENIGN SACRAL TUMORS}

\section{Surgical Approaches}

The general approaches to sacral tumors include anterior approaches and posterior approaches. ${ }^{17}$ Anterior approaches include either the transabdominal or retroperitoneal path, whereas posterior approaches involve sacral laminectomy. A third much less common route is the perineal approach for very distal sacral tumors.

Goals regarding surgery include maximal resection and preservation of neurological function. Preserving both S-1 nerve roots is important for maintaining the patient's normal gait and foot plantar flexion. Patients with bilater-

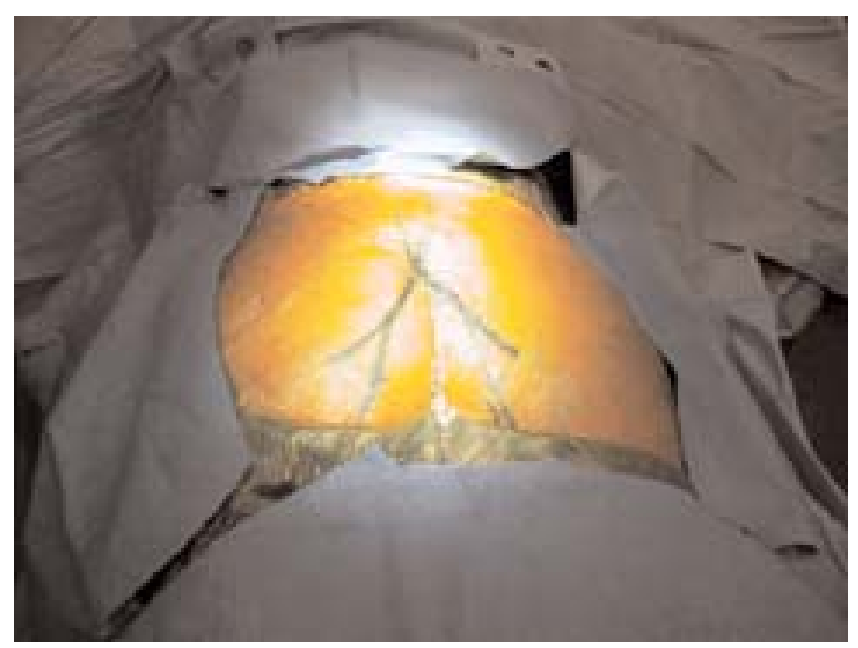

Fig. 2. Photograph showing patient positioning and surgical incision in preparation for resection of a sacral mass. 


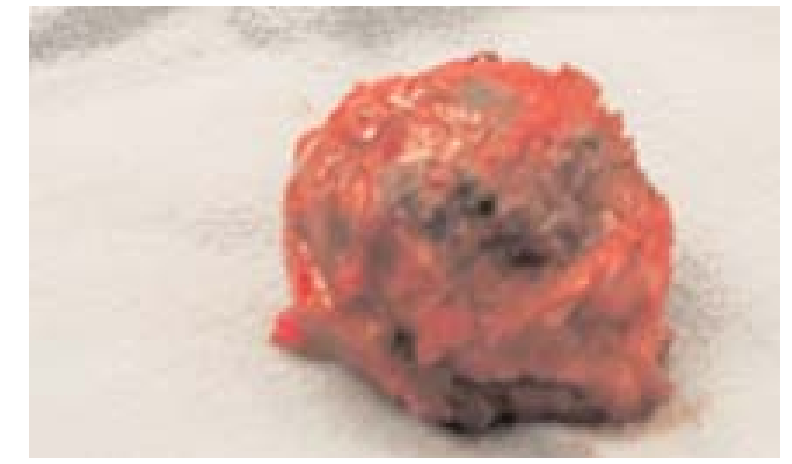

Fig. 3. Photograph displaying the resected tumor mass after a partial sacrectomy.

ally preserved S-2 nerve roots will retain bowel and bladder function, although in those in whom only one remaining S-2 nerve root remains bowel and bladder function will likely be lost. Resection of the sacrum caudal to S-1 does not require reconstruction. If $\mathrm{S}-1$ is compromised, reconstruction to stabilize the lumbar spine and ilium is required.

Combining an anterior and posterior approach is sometimes necessary. Gokaslan, et al., ${ }^{7}$ have recommended a two-staged resection for large aggressive sacral tumors to achieve complete removal. The first stage I involves an anterior approach to free the anterior tumor from pelvic, viscera, and blood vessels. Stage II involves a posterior sacrectomy, en bloc tumor resection, and sacral reconstruction.

\section{Sacral Instrumentation}

After removal of tumors that disrupt the L5-S1 disc space, reconstruction and placement of the lumbar, sacral, and the pelvic instrumentation are usually required. Tumor destruction of the sacroiliac joint requires extension of instrumentation to include the pelvis. Several techniques for reconstruction of the sacrum have been described, including the Galveston method..$^{7,19}$

Lumboiliac instrumentation includes using two L-shaped rods, which are attached to pedicle screws in the lumbar spine. ${ }^{8}$

\section{SUMMARY}

Benign sacral tumors are rare. They usually arise from either the sacral bone or from the neural compartment. Sacral tumors often grow to very large sizes before presenting clinically. They are difficult to treat surgically because of their large size. Most patients can be managed effectively with a combination of surgery and adjunctive treatment.

\section{References}

1. Abernathey CD, Onofrio BM, Scheithauer B, et al: Surgical management of giant sacral schwannomas. J Neurosurg 65: 286-295, 1986
2. Biagini R, Orsini U, Demitri S, et al: Osteoid osteoma and osteoblastoma of the sacrum. Orthopedics 24:1061-1064, 2001

3. Boretz RS, Lonner BS: Atypical presentation of an osteoid osteoma in a child. Am J Orthop 31:347-348, 2002

4. Dick HM, Bigliani LU, Michelsen WJ, et al: Adjuvant arterial embolization in the treatment of benign primary bone tumors in children. Clin Orthop 139:133-141, 1979

5. Dominguez J, Lobato RD, Ramos A, et al: Giant intrasacral schwannomas: report of six cases. Acta Neurochir 139: 954-960, 1997

6. Feldenzer JA, McGauley JL, McGillicuddy JE: Sacral and presacral tumors: problems in diagnosis and management. Neurosurgery 25:884-891, 1989

7. Gokaslan ZL, Romsdahl MM, Kroll SS, et al: Total sacrectomy and Galveston L-rod reconstruction for malignant neoplasms. Technical note. J Neurosurg 87:781-787, 1997

8. Kuklo TR, Bridwell KH, Lewis SJ, et al: Minimum 2-year analysis of sacropelvic fixation and L5-S1 fusion using S1 and iliac screws. Spine 26:1976-1983, 2001

9. Lam CH, Nagib MG: Nonteratomatous tumors in the pediatric sacral region. Spine 27:E284-E287, 2002

10. Lin PP, Guzel VB, Moura MF, et al: Long-term follow-up of patients with giant cell tumor of the sacrum treated with selective arterial embolization. Cancer 95:1317-1325, 2002

11. Marcove RC, Sheth DS, Brien EW, et al: Conservative surgery for giant cell tumors of the sacrum. The role of cryosurgery as a supplement to curettage and partial excision. Cancer 74: 1253-1260, 1994

12. O'Neill OR, Piatt JH Jr, Mitchell P, et al: Agenesis and dysgenesis of the sacrum: neurosurgical implications. Pediatr Neurosurg 22:20-28, 1995

13. Ozaki T, Halm H, Hillmann A, et al: Aneurysmal bone cysts of the spine. Arch Orthop Trauma Surg 119:159-162, 1999

14. Papagelopoulos PJ, Choudhrury SN, Frassica FJ, et al: Treatment of aneurysmal bone cysts of the pelvis and sacrum. J Bone Joint Surg Am 83:1674-1681, 2001

15. Pogoda P, Linhart W, Priemel M, et al: Aneurysmal bone cysts of the sacrum. Clinical report and review of the literature. Arch Orthop Trauma Surg 123:247-251, 2003

16. Popuri R, Davies AM: MR imaging features of giant presacral schwannomas: a report of four cases. Eur Radiol 12: 2365-2369, 2002

17. Raque GH Jr, Vitaz TW, Shields CB: Treatment of neoplastic diseases of the sacrum. J Surg Oncol 76:301-307, 2001

18. Rescorla FJ, Sawin RS, Coran AG, et al: Long-term outcome for infants and children with sacrococcygeal teratoma: a report from the Children's Cancer Group. J Pediatr Surg 33: 171-176, 1998

19. Shikata J, Yamamuro T, Kotoura Y, et al: Total sacrectomy and reconstruction for primary tumors. Report of two cases. J Bone Joint Surg Am 70:122-125, 1988

20. Turcotte RE, Sim FH, Unni KK: Giant cell tumor of the sacrum. Clin Orthop 291:215-221, 1993

21. Whalen TV Jr, Mahour GH, Landing BH, et al: Sacrococcygeal teratomas in infants and children. Am J Surg 150:373-375, 1985

Manuscript received July 2, 2003.

Accepted in final form July 17, 2003.

Address reprint requests to: Praveen V. Mummaneni, M.D., Department of Neurosurgery, Emory University, Crawford Long Hospital, 550 Peachtree St., Suite 806, Atlanta, GA 30308. email: praveen_mummaneni@emoryhealthcare.org. 